\title{
MIXED-SIGNAL GRADIENT FLOW BEARING ESTIMATION
}

\author{
Milutin Stanacevic and Gert Cauwenberghs \\ ECE Department, Johns Hopkins University, Baltimore, MD 21218, USA \\ E-mail: \{miki,gert $\} @ j h u . e d u$
}

\begin{abstract}
A mixed-signal architecture for estimating the 3-D direction cosines of a broadband traveling wave impinging on an array of four sensors is presented. The architecture implements gradient flow, which converts the problem of resolving time delays between sensor observations into the problem of estimating relative amplitudes of spatial and temporal derivatives over the array. Direction cosines of the source are obtained through least-squares adaptation on the derivative signals. Least-squares adaptive cancelation of commonmode leakthrough and correlated double sampling in finite-difference derivative estimation reduce common-mode offsets and $1 / f$ noise for increased differential sensitivity. Experimental results from an integrated circuit for acoustic localization demonstrate decimation of time delays with $5 \mu$ s resolution at $2 \mathrm{kHz}$ sampling rate.
\end{abstract}

\section{INTRODUCTION}

Continued trends towards miniaturization of integrated sensors pose the challenge of formulating source localization algorithms that perform robustly with sub-wavelength dimensions of the sensor array [1]. It is well known that the precision of delay-based bearing estimation degrades with shrinking dimensions (aperture) of the sensor array [2]. Time-difference of arrival estimation techniques based on cross-correlation of the signals [3] require high oversampling ratios for estimating small time delays [4].

Gradient flow [5] avoids the problem of estimating small time delays between sensor observations by relating amplitudes of spatial and temporal gradients in the signal across the sensor array. The idea of wavefront sensing in space for localizing sound was first introduced by Blumlein in the 1930s [6]. The direction of a traveling wave can be inferred directly by sensing spatial differentials on a sub-wavelength scale, as manifested in living biological [7, 8] and biomimetic MEMS/VLSI systems [9].

Section 2 presents the gradient flow approach to localizing a single source in the free field. Gradients in time and space are estimated using finite differences on an array of four sensors in the plane. Section 3 presents a mixed-signal architecture implementing gradient flow, including least-squares adaptation for commonmode rejection and bearing estimation. Experimental results from a fabricated prototype are presented in Section 4.

\section{GRADIENT FLOW LOCALIZATION}

A traveling wave emitted by a source is observed over a distribution of sensors in space, which here we consider to be discrete but

This work was partly supported by ONR N00014-99-1-0612, ONR/DARPA N00014-00-C-0315 and N00014-00-1-0838. which could be continuous. We define $\tau(\mathbf{r})$ as the time lag between the wavefront at point $\mathbf{r}$ and the wavefront at the center of the array, i.e., the propagation time $\tau(\mathbf{r})$ is referenced to the center of the array.

For an integrated MEMS or VLSI array with dimensions typically smaller than $1 \mathrm{~cm}$, the the distance from the source is much larger than the dimensions of the sensor array, and the far-field approximation is a sensible approximation. In the far field, the wavefront delay $\tau(\mathbf{r})$ is approximately linear in the projection of $\mathbf{r}$ on the unit vector $\mathbf{u}$ pointing towards the source,

$$
\tau(\mathbf{r}) \approx \frac{1}{c} \mathbf{r} \cdot \mathbf{u}
$$

where $c$ is the speed of (acoustic or electromagnetic) wave propagation.

Let $x(\mathbf{r}, t)$ be the signal picked up by a sensor at position $\mathbf{r}$. As one special case we will consider a two-dimensional array of sensors, with position coordinates $p$ and $q$ so that $\mathbf{r}_{p q}=p \mathbf{r}_{1}+q \mathbf{r}_{2}$ with orthogonal vectors $\mathbf{r}_{1}$ and $\mathbf{r}_{2}$ in the sensor plane. In the farfield approximation (1), the sensor observations of the source are advanced in time by $\tau_{p q}=p \tau_{1}+q \tau_{2}$, where

$$
\begin{aligned}
\tau_{1} & =\frac{1}{c} \mathbf{r}_{1} \cdot \mathbf{u} \\
\tau_{2} & =\frac{1}{c} \mathbf{r}_{2} \cdot \mathbf{u}
\end{aligned}
$$

are the inter-time differences (ITD) of source between adjacent sensors on the grid along the $p$ and $q$ place coordinates, respectively. Knowledge of the angle coordinates $\tau_{1}$ and $\tau_{2}$ uniquely determines, through (2), the direction vector $\mathbf{u}$ along which source impinges the array, in reference to the $\{p, q\}$ plane.

The signal observed at sensor with position coordinates $p$ and $q$ can be expressed as

$$
x_{p q}(t)=s\left(t+\tau_{p q}\right)+n_{p q}(t)
$$

where $n_{p q}(t)$ represent additive noise in the sensor observations. A gradient flow formulation is obtained by evaluating spatial gradients of $x_{p q}$ along the $p$ and $q$ position coordinates, around the origin $p=q=0$ :

$$
\begin{aligned}
\xi_{i j}(t) & \left.\equiv \frac{\partial^{i+j}}{\partial^{i} p \partial^{j} q} x_{p q}(t)\right|_{p=q=0} \\
& =\left(\tau_{1}\right)^{i}\left(\tau_{2}\right)^{j} \frac{d^{i+j}}{d^{i+j} t} s(t)+\nu_{i j}(t),
\end{aligned}
$$

where $\nu_{i j}$ are the corresponding spatial derivatives of the sensor noise $n_{p q}$ around the center. Taking spatial derivatives $\xi_{i j}$ of order $i+j \leq k$, and differentiating $\xi_{i j}$ to order $k-(i+j)$ in time 
yields a number of different linear observations in the $k$ th-order time derivatives of the signals $s$. The advantage of this technique is that it effectively reduces the problem of directly estimating time delays to linear regression of the derivative signals.

As an example, consider the first-order case $k=1$, corresponding to (3):

$$
\begin{aligned}
& \xi_{00}(t)=s(t)+\nu_{00}(t), \\
& \xi_{10}(t)=\tau_{1} \dot{s}(t)+\nu_{10}(t), \\
& \xi_{01}(t)=\tau_{2} \dot{s}(t)+\nu_{01}(t) .
\end{aligned}
$$

By time-differentiating the expression for $\xi_{00}$, the problem of estimating time delays converts to standard linear regression

$$
\begin{aligned}
& \xi_{10}(t) \approx \tau_{1} \dot{\xi}_{00}(t) \\
& \xi_{01}(t) \approx \tau_{2} \dot{\xi}_{00}(t)
\end{aligned}
$$

in the unknown delays $\tau_{1}$ and $\tau_{2}$. From least-square estimates of the time delays, we can directly obtain estimates of azimuth angle $\theta$ and elevation angle $\phi$ angle according to (2):

$$
\begin{aligned}
\tau_{1} & =\frac{1}{c}\left|\mathbf{r}_{1}\right| \cos \theta \sin \phi \\
\tau_{2} & =\frac{1}{c}\left|\mathbf{r}_{2}\right| \sin \theta \sin \phi
\end{aligned}
$$

An interesting observation is that the estimate of azimuth angle is independent of the speed of sound as it involves spatial gradients only; estimation of the elevation angle on the other hand requires knowledge of the speed of sound in relating spatial and temporal derivatives. The estimate of azimuth angle can be obtained simply from the ratio of delay estimates $\tau_{1}$ and $\tau_{2}$ :

$$
\hat{\theta}=\arctan \frac{\tau_{1}}{\tau_{2}}
$$

In the case of multiple sources, the observed sensor signals contain mixtures of delayed source signals, which gradient flow converts to linear mixtures of temporal derivatives of the source signals. Under the assumptions that the source signals are independent, this formulation is equivalent to standard independent component analysis (ICA), and a number of approaches exist for solving this problem. An interesting corrolary is that the mixing coefficients obtained from ICA on gradient flow transformed sensor data directly yield the angles of incoming source waves relative to the array, performing blind separation and localization of multiple sources at once. Details are given in a precursor of this paper [5].

\section{SYSTEM OVERVIEW}

The system block diagram implementing gradient flow for bearing estimation is shown in Figure 1. The differential amplification block approximates spatial and temporal gradients by computing finite differences on a planar grid of four sensors. Common-mode error correction of the first-order spatial gradients compensates for gain mismatch between sensors observations. Using temporal derivative and first-order spatial gradients estimates of time delays $\tau_{1}$ and $\tau_{2}$ are obtained.

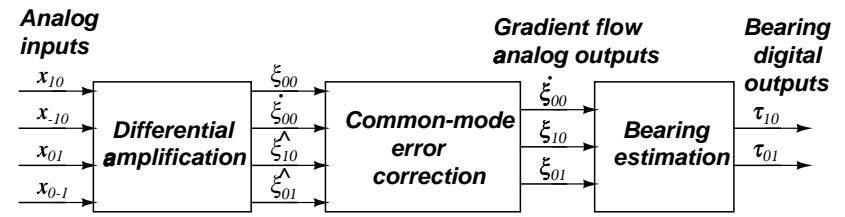

Figure 1: System diagram

\subsection{Spatial and temporal derivative sensing}

The proposed gradient flow technique requires computation of temporal derivative and first-order spatial gradients along $p$ and $q$ directions of the signal impinging on the sensor array. Estimates of $\xi_{00}, \xi_{10}$ and $\xi_{01}$ are obtained by finite-difference gradient approximation on a grid, using a planar array of four sensors:

$$
\begin{aligned}
& \xi_{00} \approx \frac{1}{4}\left(x_{-1,0}+x_{1,0}+x_{0,-1}+x_{0,1}\right) \\
& \xi_{10} \approx \frac{1}{2}\left(x_{1,0}-x_{-1,0}\right) \\
& \xi_{01} \approx \frac{1}{2}\left(x_{0,1}-x_{0,-1}\right)
\end{aligned}
$$

Computation of the gradients is implemented using sampled-data switched-capacitor (SC) circuits. The advantage of this realization is application of correlated-double sampling (CDS), a technique that strongly reduces common-mode offsets and $1 / f$ noise [10]. The spatial gradients are computed in fully differential mode, to provide increased clock and supply feedthrough rejection.

The common-mode component $\xi_{00}$ is computed in the following manner

$$
\begin{aligned}
\xi_{00}^{+}[n]= & \frac{1}{8}\left(x_{10}\left[n-\frac{1}{2}\right]+x_{-10}\left[n-\frac{1}{2}\right]\right. \\
& \left.+x_{01}\left[n-\frac{1}{2}\right]+x_{0-1}\left[n-\frac{1}{2}\right]\right) \\
\xi_{00}^{-}[n]= & -\frac{1}{8}\left(x_{10}[n]+x_{-10}[n]+x_{01}[n]+x_{0-1}[n]\right),
\end{aligned}
$$

and the computation is illustrated in Figure 2. Positive contribution $\xi_{00}^{+}$to $\xi_{00}$ represents the estimate of average signal at time instance $n T-\frac{T}{2}$, while negative contribution $\xi_{00}^{-}$represents the inverted estimate at time instance $n T$. The difference between both contributions signals produces an unbiased estimate of $\xi_{00}$ centered at time $n T-\frac{T}{4}$, by taking the average of the estimates at times $n T-\frac{T}{2}$ and $n T$.

An estimate for the temporal derivative signal $\dot{\xi}_{00}$ centered at same time instance $n T-\frac{T}{4}$ is computed in similar manner, by taking the difference of estimates $\xi_{00}$ at time instances $n T-\frac{T}{2}$ and $n T$

$$
\begin{aligned}
\dot{\xi}_{00}^{+}[n]= & 0 \\
\dot{\xi}_{00}^{-}[n]= & -\frac{1}{8}\left(x_{10}[n]+x_{-10}[n]+x_{01}[n]+x_{0-1}[n]\right) \\
& +x_{10}\left[n-\frac{1}{2}\right]+x_{-10}\left[n-\frac{1}{2}\right] \\
& +x_{01}\left[n-\frac{1}{2}\right]+x_{0-1}\left[n-\frac{1}{2}\right] .
\end{aligned}
$$

The temporal derivative computation is illustrated in Figure 3.

The first order spatial gradient in $p$ direction $\xi_{10}$, again centered at time $n T-\frac{T}{4}$, is computed by differencing estimates of 

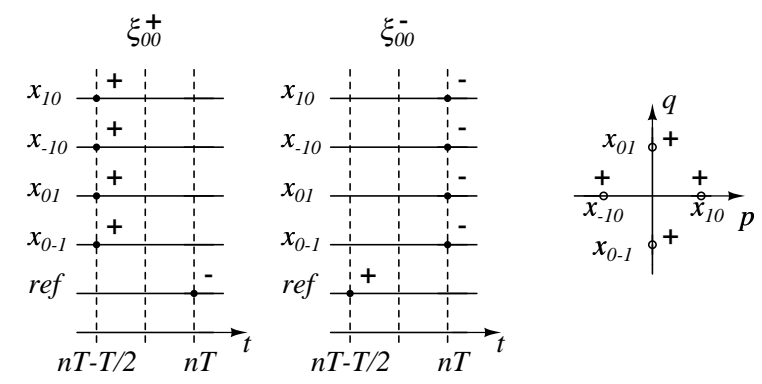

Figure 2: Evaluation of common-mode (average) signal $\xi_{00}$.
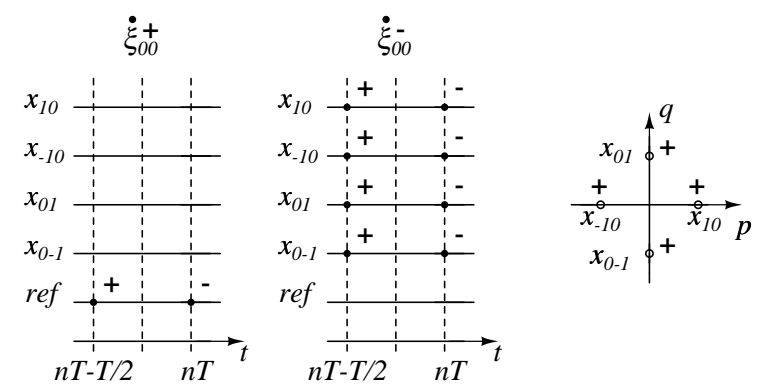

Figure 3: Evaluation of temporal derivative common-mode signal $\dot{\xi}_{00}$.

$\xi_{10}$ at time instances $n T-\frac{T}{2}$ and $n T$

$$
\begin{aligned}
& \xi_{10}^{+}[n]=\frac{1}{4}\left(x_{10}\left[n-\frac{1}{2}\right]-x_{-10}[n]\right) \\
& \xi_{10}^{-}[n]=\frac{1}{4}\left(x_{-10}\left[n-\frac{1}{2}\right]-x_{10}[n]\right)
\end{aligned}
$$

The computation is illustrated in Figure 4. First order spatial gradient in $q$ direction, $\xi_{01}$, is computed in the same fashion.

As an example, switched-capacitor realization of the computation of the first-order spatial gradient is shown in Figure 5.

\subsection{Common-mode suppression}

Before bearing estimation of direction cosines using temporal and spatial gradients, common mode offset correction is performed on the estimated spatial gradients. Common mode offsets arise from gain mismatch errors in the sensors and in the circuits computing the gradients, and can be represented to first order as

$$
\begin{aligned}
\hat{\xi}_{10} & \approx \xi_{10}+\varepsilon_{1} \xi_{00} \\
& \approx \tau_{1} \dot{s}(t)+\varepsilon_{1} s(t)
\end{aligned}
$$

From second order statistics, the correlation between any signal and its time-derivative is zero

$$
\mathrm{E}[\dot{s}(t) s(t)]=0
$$

which implies that the correlation between common-mode and gradient variable is also zero

$$
\mathrm{E}\left[\xi_{00} \xi_{10}\right]=0 .
$$

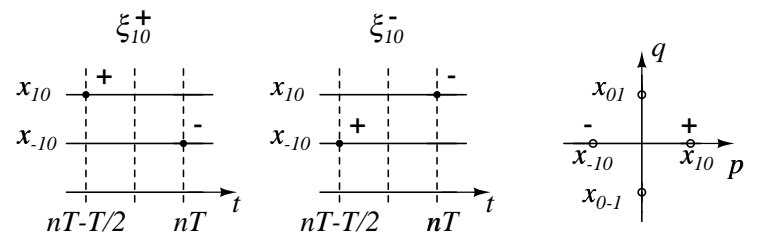

Figure 4: Evaluation of first-order spatial gradient along $p$ direction, $\xi_{10}$.

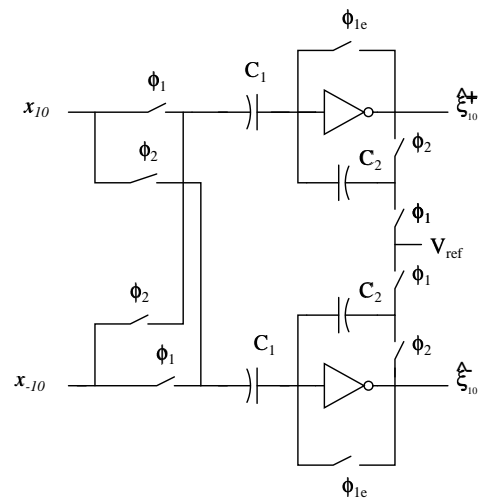

Figure 5: Switched-capacitor differential realization of the firstorder spatial gradient $\xi_{10}$.

The spatial gradient with eliminated systematic common-mode error is then estimated using

$$
\xi_{10} \approx \hat{\xi}_{10}-\frac{\mathrm{E}\left[\xi_{00} \hat{\xi}_{10}\right]}{\mathrm{E}\left[\xi_{00}^{2}\right]} \xi_{00} .
$$

To estimate $\varepsilon_{1}$, digital sign-sign LMS (SS-LMS) adaptation is used. $\varepsilon_{1}$ is updated using a 12-bit digital counter and it is represented in two's complement

$$
\begin{aligned}
\varepsilon_{1}^{+}[n+1]= & \varepsilon_{1}^{+}[n] \\
& +\operatorname{sgn}\left(\xi_{10}^{+}[n]-\xi_{10}^{-}[n]\right) \operatorname{sgn}\left(\xi_{00}^{+}[n]-\xi_{00}^{-}[n]\right) \\
\varepsilon_{1}^{-}[n+1]= & 2^{12}-1-\varepsilon_{1}^{+}[n+1]
\end{aligned}
$$

The 8 most significant bits are presented to a multiplying D/A capacitor array to construct the LMS error signal, in this case $\xi_{10}$,

$$
\begin{aligned}
& \xi_{10}^{+}[n]=\hat{\xi}_{10}^{+}[n]-\left(\varepsilon_{1}^{+} \xi_{00}^{+}[n]+\varepsilon_{1}^{-} \xi_{00}^{-}[n]\right) \\
& \xi_{10}^{-}[n]=\hat{\xi}_{10}^{-}[n]-\left(\varepsilon_{1}^{-} \xi_{00}^{+}[n]+\varepsilon_{1}^{+} \xi_{00}^{-}[n]\right) .
\end{aligned}
$$

The range of common-mode error correction is designed to compensate for at most 5 percent of the average signal in the spatial gradients.

\subsection{Bearing estimation}

For bearing time-delay estimation, digital SS-LMS differential online adaptation is used in implementation similar to the one used for common-mode error correction. Bearing estimates are represented as 12-bit values in two's complement

$$
\begin{aligned}
\tau_{1}^{+}[n+1]= & \tau_{1}^{+}[n] \\
& +\operatorname{sgn}\left(e_{10}^{+}[n]-e_{10}^{-}[n]\right) \operatorname{sgn}\left(\xi_{10}^{+}[n]-\xi_{10}^{-}[n]\right) \\
\tau_{1}^{-}[n+1]= & 2^{12}-1-\tau_{1}^{+}[n+1]
\end{aligned}
$$




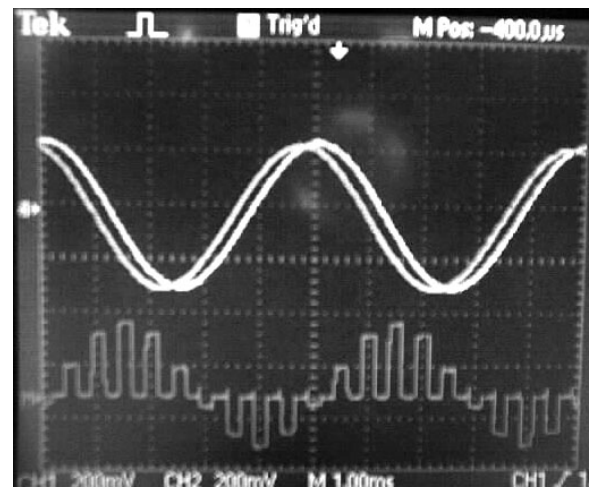

Figure 6: Computation of spatial gradient. Top: delayed sine wave inputs $x_{10}$ and $x_{-10}$. Bottom: differenced gradient signal $\xi_{10}=$ $\xi_{10}^{+}-\xi_{10}^{-}$.

with 8 most significant bits used for computation of LMS error signal

$$
\begin{aligned}
& e_{10}^{+}[n]=\xi_{10}^{+}[n]-\left(\tau_{1}^{+} \dot{\xi}_{00}^{+}[n]+\tau_{1}^{-} \dot{\xi}_{00}^{-}[n]\right) \\
& e_{10}^{-}[n]=\xi_{10}^{-}[n]-\left(\tau_{1}^{-} \dot{\xi}_{00}^{+}[n]+\tau_{1}^{+} \dot{\xi}_{00}^{-}[n]\right) .
\end{aligned}
$$

One characteristic of the discrete-time implementation is that the ratio of computed spatial and temporal derivative is proportional to sampling frequency of the systems. If for example the sampling frequency is $16 \mathrm{kHz}$, the minimum time delay that can be resolved is $0.375 \mu \mathrm{s}$.

\section{RESULTS}

The architecture was integrated on a single chip fabricated in $0.5 \mu \mathrm{m}$ CMOS technology. To demonstrate the gradient flow bearing estimation, the following experimental setup was used. The sampling frequency of the system was $2 \mathrm{kHz}$. A sinewave signal of $200 \mathrm{~Hz}$ was fed to the inputs which represent the signal observed at sensors $x_{10}$ and $x_{01}$. The sinewave was passed through low-pass $R C$ filter, which introduces a delay. The delayed sinewave was fed to chip input representing sensor signals $x_{-10}$ and $x_{0-1}$. Figure 6 illustrates computation of the spatial gradient for a given delay. By varying the resistance, the delay is changed. Amplitude differences between the two sinewaves are compensated on-chip by common-mode cancelation. The delay was varied from $20 \mu$ s to $400 \mu \mathrm{s}$, in $20 \mu \mathrm{s}$ increments. The chip outputs bit-serial digital estimates of $\tau_{1}$ and $\tau_{2}$, obtained directly from the bearing registers (19). The recorded delay $\tau_{1}$ as a function of actual delay is shown in Figure 7.

\section{CONCLUSION}

We presented a robust mixed-signal architecture for gradient flow bearing estimation, that combats problems of gain mismatch and $1 / f$ noise originating from the sensor array and preamplifiers. The principle was demonstrated on an experimental prototype with 4 sensor analog inputs and 2 digital estimated time delay outputs. The chip can potentially be integrated with the sensor array, for a small, compact, battery-operated "smart" sensor applications in surveillance and hearing aids.

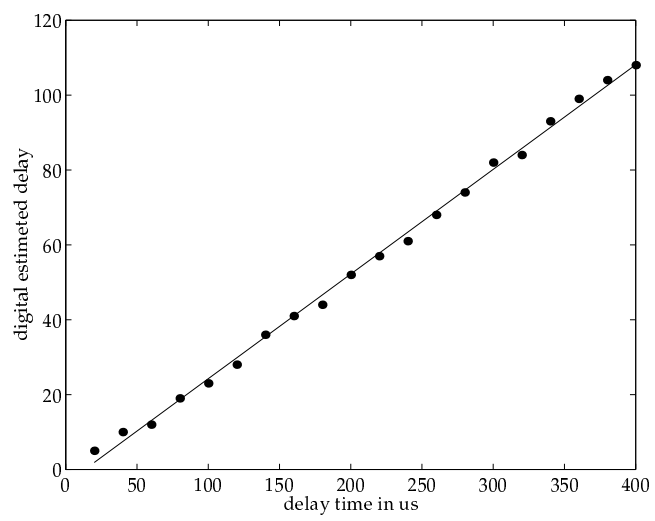

Figure 7: Experimental results

\section{REFERENCES}

[1] P. Julian, A. G. Andreou, L. Riddle, S. Shamma, and G. Cauwenberghs, "A comparison of methods for sound localization," in Proc. IEEE Int. Symp. on Circuits and Systems (ISCAS'2003), Bangkok, Thailand, 2003.

[2] S. Haykin, Adaptive Filter Theory, Prentice-Hall, 2nd edition, 1991.

[3] C.H. Knapp and G.C. Carter, "The generalized correlation method for estimation of time delay," IEEE Trans. on Acoustics, Speech, and Signal Processing, vol. 24, no. 4, pp. 320327, 1976.

[4] P. Julian, A. G. Andreou, P. Mandolesi, and D. Goldberg, "A low-power CMOS integrated circuit for bearing estimation," in Proc. IEEE Int. Symp. on Circuits and Systems (ISCAS'2003), Bangkok, Thailand, 2003.

[5] G. Cauwenberghs, M. Stanacevic, and G. Zweig, "Blind broadband source localization and separation in miniature sensor arrays," in Proc. IEEE Int. Symp. Circuits and Systems (ISCAS'2001), Sydney, Australia, 2001, vol. 3, pp. 193 $-196$.

[6] A.D. Blumlein, "Improvements in and relating to soundtransmission, sound-recording and sound-reproducing systems," British Patent, , no. 394325, 1933.

[7] D. Robert, R.N. Miles, and R.R. Hoy, "Tympanal hearing in the sarcophagid parasitoid fly emblemasoma sp.: the biomechanics of directional hearing," J. Experimental Biology, vol. 202, pp. 1865-1876, 1999.

[8] J.A. Simmons, M.J. Ferragamo, and C.F. Moss, "Echo-delay resolution in sonar images of the big brown bat, eptesicusfuscus," Proc. Nat. Academy Sciences USA, vol. 95, no. 21, pp. 12647-12652, Oct 1998.

[9] A.G. Andreou, D.H. Goldberg, E. Culurciello, M. Stanacevic, G. Cauwenberghs, and L. Riddle, "Heterogeneous integration of biomimetic acoustic microsystems," in Proc. IEEE Int. Symp. Circuits and Systems (ISCAS'2001), Sydney, Australia, May 6-9 2001.

[10] C.C. Enz and G.C. Temes, "Circuit tecniques for reducing the effects of op-amp imperfections: Autozeroing, correlated double sampling, and chopper stabilization," IEEE Proceedings, vol. 84, no. 11, pp. 1584-1614, 1996. 